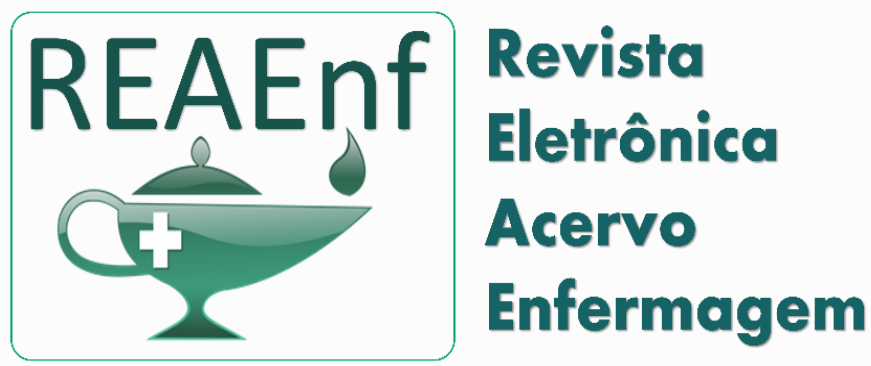

REVISÃO BIBLIOGRÁFICA

Recebido em: 12/2020

Aceito em: 1/2021

Publicado em: 2/2021

\title{
Saúde mental dos estudantes universitários
}

\author{
Mental health of university students
}

\section{Salud mental de estudiantes universitários}

Maria Eduarda Alves da Silva ${ }^{1 *}$, Rayza Rodrigues dos Santos ${ }^{1}$, Roberta Vanyhellen de Jesus Medeiros $^{1}$, Sandiely Lorrainy de Carvalho Souza ${ }^{1}$, Dagmar Fonseca Souza ${ }^{1}$, Deuzileni Pereira Vieira Ferreira ${ }^{1}$.

\begin{abstract}
Resumo: O objetivo deste estudo é analisar a produção de artigos científicos no período de 2010 a 2020 referentes aos principais problemas de saúde mental apresentados por estudantes universitários e o que os leva a desenvolvêlos. Trata-se de uma revisão integrativa da literatura sobre o tema proposto. Para obtenção dos dados, foram utilizadas as bases de dados Scientific Eletronic Library online (SCIELO), Biblioteca Virtual de Saúde (BVS), Plataforma de educação básica (CAPES) e PubMed. Os resultados indicaram que de fato a vida acadêmica interfere na saúde mental dos estudantes e estes são identificados como um grupo vulnerável e de risco nas categorias de ansiedade, depressão e uso de drogas, e essas condições são pontos fundamentais para o desenvolvimento de ações em saúde mental. É necessário que a instituição de ensino superior desenvolva estratégias para prevenir agravos a saúde mental de seus discentes, para que assim possam concluir o ensino superior com saúde mental estável.
\end{abstract}

Palavras-chave: Ansiedade, Depressão, Estudantes universitários.

\begin{abstract}
The objective of this study is to analyze the production of scientific articles in the period from 2010 to 2020 regarding the main mental health problems presented by university students and what leads them to develop them. It is an integrative review of the literature on the proposed theme. The Scientific Electronic Library online (SCIELO), Virtual Health Library (VHL), Basic Education Platform (CAPES) and PubMed databases were used to obtain the data. The results indicated that in fact academic life interferes with students' mental health and they are identified as a vulnerable and risk group in the categories of anxiety, depression and drug use, and these conditions are fundamental points for the development of mental health actions. It is necessary for the higher education institution to develop strategies to prevent aggravation to the mental health of its students, so that they can conclude higher education with stable mental health.
\end{abstract}

Keywords: Anxiety, Depression, University students.

Resumen: El objetivo de este estudio es analizar la producción de artículos científicos en el período de 2010 a 2020 sobre los principales problemas de salud mental que presentan los estudiantes universitarios y lo que los lleva a desarrollarlos. Es una revisión integral de la literatura sobre el tema propuesto. Para obtener los datos se utilizaron las bases de datos de la Biblioteca Científica Electrónica en línea (SCIELO), la Biblioteca Virtual de Salud (BVS), la Plataforma de Educación Básica (CAPES) y PubMed. Los resultados indicaron que, de hecho, la vida académica interfiere con la salud mental de los estudiantes y se los identifica como un grupo vulnerable y de riesgo en las categorías de ansiedad, depresión y consumo de drogas, y estas condiciones son puntos fundamentales para el desarrollo de acciones de salud mental. Es necesario que la institución de enseñanza superior elabore estrategias para evitar el agravamiento de la salud mental de sus estudiantes, de modo que puedan concluir la enseñanza superior con una salud mental estable.

Palabras clave: Ansiedad, Depresión, Estudiantes universitarios.

1 Universidade do Estado do Pará (UEPA), Conceição do Araguaia - PA.

*E-mail: maria_dudaenf@outlook.com 


\section{INTRODUÇÃO}

A Organização Mundial de Saúde (1946), define saúde como: "um estado de completo bem-estar físico, mental e social, e não consiste apenas na ausência de doença ou de enfermidade", ou seja, a saúde mental é indispensável para o bem-estar geral dos indivíduos e da sociedade. No entanto, procurar manter tudo equilibrado sem deixar nenhuma delas de lado, no dia a dia, é complicado, devido a rotina corrida que leva um universitário. Portanto, devido à falta de tempo para se cuidar acaba deixando uma ou mais delas de lado, sem poder buscar o equilíbrio (BRAGA ALS, et al., 2017).

O ingresso na Universidade é caracterizado por diversas mudanças, sendo necessário um processo de adaptação, pois o ambiente acadêmico é repleto de normas, metodologias, grupos e pessoas desconhecidas, sendo assim indispensável que o estudante crie um perfil universitário. Porém, esse processo pode gerar angústias, conflitos, ansiedade, e essa nova realidade coloca o universitário em um estado de vulnerabilidade, aumentando as chances de quadros psicopatológicos e dificuldades no desenvolvimento pessoal e profissional (CASTRO VR, 2017).

Os universitários, na maior parte de seu tempo estão focados em trabalhos, provas, monitorias, estágios, entre outras atividades, e deixam de lado coisas que antes eram importantes, tais como, família, amigos, namorado, religião, academia, atividades esportivas e de recreação. Quando enfim conseguem um tempo para fazer alguma atividade que não esteja relacionada à universidade, já estão no limite do estresse, com a saúde mental muito debilitada (BRAGA ALS, et al., 2017).

Neste sentido, convém destacar que uma psicopatologia é uma alteração dos processos cognitivos e afetivos do desenvolvimento, que se traduz em perturbações no nível de raciocínio, comportamento, compreensão da realidade e da adaptação às condições da vida. Em situações de saúde onde o indivíduo apresenta sintomas de depressão, ansiedade em intensidade suficiente para que as atividades diárias sofram interferências, sem diagnóstico formal, dá-se o nome de transtorno mental comum (SADOCK BJ, et al., 2016; GOMES CFM, et al., 2020).

Transtornos mentais comuns correlacionam-se e prejudicam as atividades diárias, afetando o desempenho acadêmico, são problemas emergentes e preocupam pela sua prevalência e seus efeitos prejudiciais à saúde dos estudantes. A prevalência de depressão em universitários, é em média 30,6\%, já na população em geral essa prevalência corresponde a 9\% (COSTA MC e MOREIRA YB, 2016; FERNANDES MA, et al., 2018).

O objetivo deste estudo é analisar a produção de artigos científicos no período de 2010 a 2020 referentes aos principais problemas de saúde mental apresentados por estudantes universitários e o que os leva a desenvolvê-los.

\section{MÉTODOS}

Este estudo é uma revisão integrativa da literatura, uma metodologia que proporciona a síntese das pesquisas disponíveis sobre determinada temática e direciona a prática fundamentando-se em conhecimento científico.

O levantamento dos trabalhos publicados foi realizado nas bases de dados, Scientific Eletronic Library online (SCIELO), Biblioteca Virtual de Saúde (BVS), Plataforma de educação básica (CAPES) e PubMed, durante o mês de outubro de 2020, utilizando os descritores: saúde mental, universitários, depressão, ansiedade e drogas. Foram realizadas pesquisas com múltiplas combinações dos descritores.

Para a consulta na literatura e seleção dos estudos, seguiram-se os critérios de inclusão ilustrados na Figura 1.

A partir da busca, foram localizadas 64 publicações referentes a temática e de acordo com os descritores estabelecidos, destes, 47 foram excluídas por não atender aos critérios de seleção, restando 17 trabalhos para constituir o banco de dados dessa pesquisa. Logo, foram extraídos os anos de publicação, títulos, fonte, autores, profissão, objetivos, métodos e resultados para construção dos quadros. 
Figura 1 - Fluxograma de critérios de inclusão e exclusão.

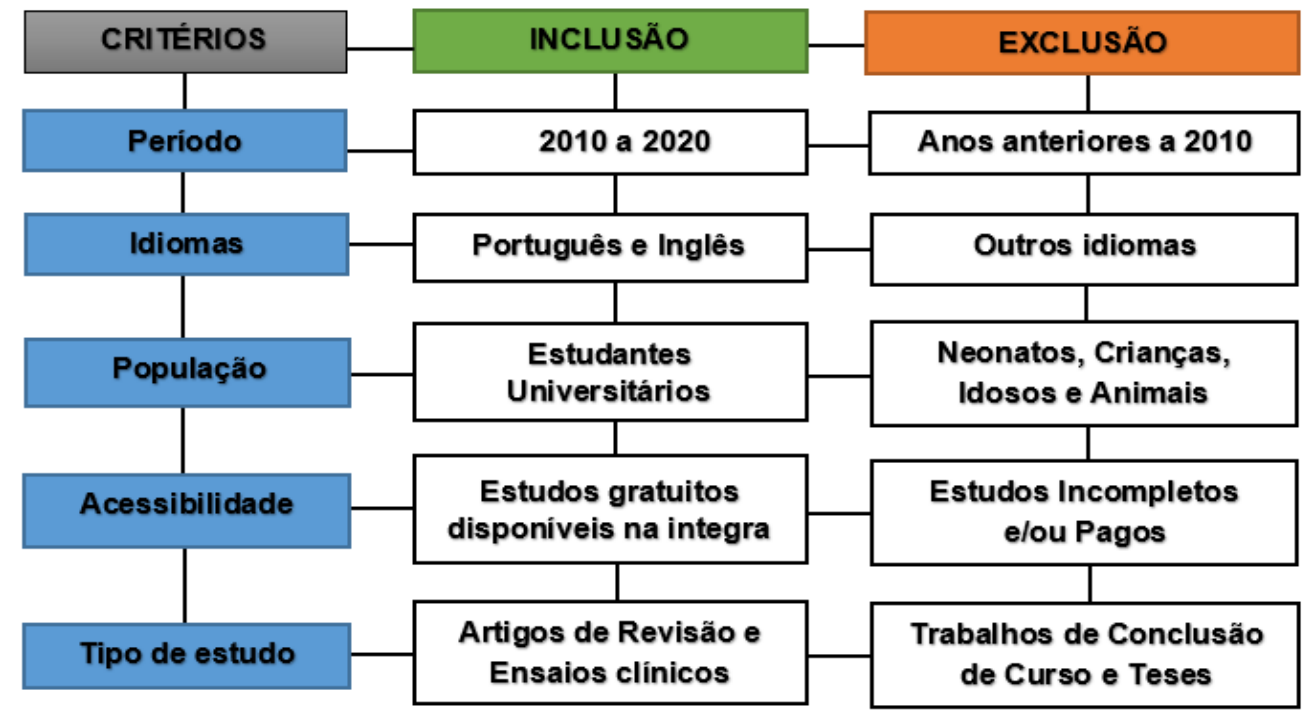

Fonte: Silva MEA, et al., 2021.

Das 64 publicações encontradas 5 foram na SciELO, 27 na BVS, 18 na PubMed e 14 na CAPES. Grande parte dos estudos estavam em idioma inglês. Assim como estudos abordando hábitos e estilos de vida dos estudantes, síndromes mentais e comportamento suicida, também foram verificados estudos sobre o comportamento do consumo de substâncias psicoativas. Após a aplicação dos critérios estabelecidos neste estudo, a presente revisão selecionou 17 artigos para constituir o banco de dados dessa pesquisa.

\section{RESULTADOS}

A pesquisa na plataforma Scielo, com a associação dos descritores: Mental health AND university AND depression AND anxiety AND drugs, resultou em 5 artigos com texto completo disponíveis, sendo 3 em português, 1 em espanhol e 1 em inglês. Desses, 4 artigos foram eleitos.

No portal regional da Biblioteca Virtual em Saúde-BVS, a busca resultou em 256 artigos com a combinação Mental health AND university AND depression AND anxiety AND drugs. Mediante os critérios de inclusão, totalizou em 27 artigos publicados entre 2010 e 2020. Selecionou-se 7 artigos pelo título, no entanto, apenas 4 foram incluídos, os demais se repetiam.

Com a busca na PubMed, utilizando a associação dos descritores Mental health AND university, resultaram 10975 artigos, e após aplicação dos critérios de inclusão e exclusão 18 artigos foram selecionados. Após leitura e exclusão de artigos repetidos, 6 foram eleitos.

No portal de periódicos CAPES, a partir da combinação de todos os descritores em português, saúde mental AND universitário AND depressão AND ansiedade AND drogas derivou 163 artigos. Ao adicionar os critérios de inclusão e exclusão procederam 14 artigos, destes, selecionou-se 3. Assim, 64 publicações foram encontradas (Tabela 1). Após a aplicação dos critérios estabelecidos foram selecionados 17 artigos para constituir o banco de dados dessa pesquisa (Quadro 1).

Tabela 1 - Artigos pesquisados e selecionados segundo os descritores e base de dados.

\begin{tabular}{cccccc}
\hline Descritores & SciELO & BVS & PubMed & CAPES & Total \\
\hline $\begin{array}{c}\text { Saúde Mental; Universitários; } \\
\text { Depressão; Ansiedade; Drogas; }\end{array}$ & 5 & 27 & 18 & 14 & 64 \\
\hline Artigos Selecionados & 4 & 4 & 6 & 3 & 17 \\
\hline
\end{tabular}

Fonte: Silva MEA, et al., 2021. 
Quadro 1 - Apresentação dos artigos selecionados para essa revisão, com Autor e Ano, Objetivos e Principais resultados.

\begin{tabular}{|c|c|c|}
\hline Autor e Ano & Objetivo & Principais Resultados \\
\hline $\begin{array}{l}\text { Angelucci LT, et } \\
\quad \text { al., } 2017\end{array}$ & $\begin{array}{c}\text { Determinar a influência do estilo de vida, sexo, idade e índice de } \\
\text { massa corporal na saúde física e psicológica de jovens } \\
\text { universitários. }\end{array}$ & $\begin{array}{l}\text { Baixos níveis de depressão e ansiedade. Hábitos inadequados na } \\
\text { dieta, atividade física e check-ups médicos. O uso de drogas é baixo. }\end{array}$ \\
\hline $\begin{array}{l}\text { Ariño DO e } \\
\text { Bardagi MP, } \\
\quad 2018\end{array}$ & $\begin{array}{l}\text { Analisar as relações entre ansiedade, depressão e stress com a } \\
\text { qualidade das vivências acadêmicas e a autoeficácia. }\end{array}$ & $\begin{array}{c}\text { Há correlação negativa entre ansiedade, stress e depressão, } \\
\text { vivências acadêmicas e autoeficácia. }\end{array}$ \\
\hline $\begin{array}{l}\text { Barbosa LNF, et } \\
\quad \text { al., } 2020\end{array}$ & $\begin{array}{l}\text { Identificar a frequência de ansiedade, depressão e o uso de } \\
\text { substâncias psicoativas em universitários. }\end{array}$ & $\begin{array}{l}\text { Ansiedade: } 28,45 \% \text { e depressão: } 16,38 \% \text { e frequência de uso de } \\
\text { substâncias em estudantes com sintomas de ansiedade. }\end{array}$ \\
\hline $\begin{array}{l}\text { Barros S e } \\
\text { Claro HG, } 2011\end{array}$ & $\begin{array}{l}\text { Representações dos alunos sobre o desenvolvimento de } \\
\text { competências na área de enfermagem em saúde mental. }\end{array}$ & $\begin{array}{l}\text { Os alunos possuem dificuldade para compreender este conteúdo e } \\
\text { conceituá-lo de forma adequada. }\end{array}$ \\
\hline $\begin{array}{l}\text { Barroso SM, et } \\
\quad \text { al., } 2019\end{array}$ & Avaliar a solidão e a depressão em universitários. & $\begin{array}{l}\text { Correlação entre solidão e depressão e dessas condições com } \\
\text { hábitos dos universitários. }\end{array}$ \\
\hline $\begin{array}{l}\text { Brandão MP, et } \\
\quad \text { al., } 2011\end{array}$ & $\begin{array}{l}\text { Avaliar a influência da vida acadêmica na saúde de estudantes } \\
\text { universitários. }\end{array}$ & $\begin{array}{l}\text { Estudantes expostos à vida acadêmica, apresentaram proporção mais } \\
\text { elevada de dislipidemia, sobrepeso e tabagismo. }\end{array}$ \\
\hline $\begin{array}{l}\text { Cardoso NF, et } \\
\text { al., } 2018\end{array}$ & $\begin{array}{l}\text { Investigar os fatores de proteção e risco relacionados à saúde } \\
\text { mental de jovens. }\end{array}$ & $\begin{array}{l}\text { Indivíduos com diagnóstico de depressão reportaram maiores níveis } \\
\text { de afetos } \\
\text { negativos, ansiedade e depressão. }\end{array}$ \\
\hline $\begin{array}{l}\text { Cecconello WW, } \\
\text { et al., } 2013\end{array}$ & $\begin{array}{l}\text { Averiguar a presença de sintomas depressivos em indivíduos com } \\
\text { FS (Fobia Social). }\end{array}$ & $\begin{array}{l}\text { Indivíduos com sintomas de FS apresentam comorbidade com } \\
\text { sintomas depressivos. }\end{array}$ \\
\hline
\end{tabular}

$\overline{\text { REAEnf/EJNC | Vol. } 9 \text { | e6228 | DOI: https://doi.org/10.25248/REAenf.e6228.2021 } \quad \text { Página } 4 \text { de } 9}$ 
Revista Eletrônica Acervo Enfermagem / Electronic Journal Nursing Collection | ISSN 2674-7189

\begin{tabular}{|c|c|c|}
\hline Autor e Ano & Objetivo & Principais Resultados \\
\hline $\begin{array}{l}\text { Dam CL, et al., } \\
2014\end{array}$ & $\begin{array}{l}\text { Determinar a prevalência de sofrimento psíquico e sua relação } \\
\text { com o engajamento acadêmico. }\end{array}$ & $\begin{array}{l}\text { Prevalência de sofrimento psíquico de } 32,2 \% \text { e correlação entre vigor } \\
\text { e sofrimento psíquico. }\end{array}$ \\
\hline $\begin{array}{l}\text { Facioli AM, et al., } \\
2020\end{array}$ & $\begin{array}{l}\text { Medir os níveis de depressão e a associação com aspectos a vida } \\
\text { acadêmica. }\end{array}$ & $19,2 \%$ apresentaram níveis de depressão moderados ou graves. \\
\hline $\begin{array}{l}\text { Flesch BD, et al., } \\
2020\end{array}$ & $\begin{array}{c}\text { Avaliar a prevalência e fatores associados ao episódio depressivo } \\
\text { maior em universitários. }\end{array}$ & $\begin{array}{l}32 \% \text { apresentou episódio depressivo maior. } \\
\text { Sexo feminino, idade, histórico familiar e morar com amigos são } \\
\text { fatores associados. }\end{array}$ \\
\hline $\begin{array}{l}\text { Leão AM, et al., } \\
\qquad 2018\end{array}$ & $\begin{array}{l}\text { Estimar a prevalência e os fatores associados à depressão e } \\
\text { ansiedade em estudantes universitários da área da saúde. }\end{array}$ & $\begin{array}{l}\text { Depressão } 28,6 \% \text { e ansiedade } 36,1 \% \text {. Estudantes menos satisfeitos } \\
\text { com o curso apresentaram mais chance de terem depressão. }\end{array}$ \\
\hline $\begin{array}{l}\text { Osse CMC e } \\
\text { Costa II, } 2011\end{array}$ & $\begin{array}{l}\text { Mapear condições psicossociais e qualidade de vida de } \\
\text { universitários da moradia estudantil da Universidade de Brasília. }\end{array}$ & $\begin{array}{l}\text { Estudantes dependentes de recursos institucionais apresentaram } \\
\text { indícios de ansiedade, depressão. }\end{array}$ \\
\hline $\begin{array}{l}\text { Rocha AMC, et } \\
\text { al., } 2020\end{array}$ & $\begin{array}{c}\text { Analisar a ocorrência de tratamento psicológico e/ou psiquiátrico } \\
\text { antes do ingresso na universidade. }\end{array}$ & $\begin{array}{l}\text { Tratamento psíquico prévio ao ingresso na universidade pode estar } \\
\text { associado ao sofrimento mental do estudante de Medicina. }\end{array}$ \\
\hline $\begin{array}{l}\text { Santos SS, et al., } \\
\qquad 2013\end{array}$ & $\begin{array}{l}\text { Comparar a opinião de estudantes de Enfermagem frente à } \\
\text { doença mental e suas práticas de cuidado. }\end{array}$ & $\begin{array}{c}\text { Os estudantes apresentaram características distintas, significativas } \\
\text { apenas em relação à benevolência. }\end{array}$ \\
\hline
\end{tabular}

Fonte: Silva MEA, et al., 2021.

$\overline{\text { REAEnf/EJNC | Vol. } 9 \text { | e6228 | DOI: https://doi.org/10.25248/REAenf.e6228.2021 Página } 5 \text { de } 9}$




\section{DISCUSSÃO}

\section{A vida acadêmica e sua influência na saúde mental dos universitários}

A universidade é um espaço importante para o desenvolvimento de vida, pois ela promove a ampliação de habilidades e competências profissionais e pessoais. Ela fornece um misto de oportunidades, interações sociais, constituindo-se como um espaço gerador de impactos positivos ou negativos para os estudantes (ARIÑO DO e BARDAGI MP, 2018; LELIS KC, et al., 2020; BRANDÃO MP, et al., 2011).

Vale ressaltar que o ingresso na universidade traz consigo também grandes mudanças para os estudantes (OSSE CMC e COSTA II, 2011; BARBOSA LNF, et al., 2020). O ritmo de vida torna-se mais intenso, a carga horária de estudos elevada, e muitas vezes ocorre o distanciamento geográfico da família, bem como as elevadas cobranças pela sociedade, instituição e pelo próprio indivíduo que podem provocar sentimentos como desapontamento, irritabilidade, preocupação e impaciência durante a graduação (ROCHA AMC, et al., 2020; LELIS KC, et al., 2020; FERNANDES MA, et al., 2018; FACIOLI AM, et al., 2020).

Esse misto de modificações e emoções que invadem a vida do jovem universitários, coincidem com um período do desenvolvimento psicossocial marcado por mudanças importantes. É o período de explorações definido como a idade das possibilidades, sendo pautado por instabilidades e reconhecidamente a fase de desenvolvimento mais auto focada: o jovem se encontra em um processo de transição complexo (OSSE CMC e COSTA II, 2011; CARDOSO HF, et al., 2018).

Essa fusão resulta em falhas de enfrentamento e/ou induzir comportamentos inadequados ou formação de sintomas não só físicos, mas também emocionais (OSSE CMC e COSTA II, 2011). Facioli AM, et al. (2020), em seu estudo, afirma que estudantes com saúde mental abalada afeta integralmente seu estado físico, mental, social e sua performance acadêmica.

Logo, Lelis KC, et al. (2020), afirma que é comum que a saúde mental dos acadêmicos esteja debilitada, diversos fatores implicam nessa condição, porém, devido a sentimentos de medo e insegurança não buscam tratamento. Vale ressaltar, que não ser excluído, não ser abandonado, não ser violentado, ser bem atendido nos serviços de saúde, ser atendido, ser reconhecido em suas necessidades é direito de todo cidadão (BARROS S e CLARO HG, 2011). Por isso, essa classe deve ser assistida mesmo quando não vão em busca de tratamento, através de apoio acadêmico, entre outros.

Resultados obtidos na pesquisa de Rocha AMC, et al. (2020), mostraram elevada prevalência de alunos com histórico de tratamento psíquico prévio ao ingresso na universidade e que também foram buscar suporte nos primeiros anos do curso. Isso pode-se explicar, através do fato de que universitários são uma população vulnerável e mais propensa a desenvolver transtornos mentais (ARINÕ DO e BARDAGI MP, 2018; BARBOSA LNF, et al., 2020). Estudos apontam que psicopatologias comuns na população universitária são problemas emergentes e preocupam pela sua prevalência e por seus efeitos deletérios à saúde dos estudantes (ROCHA AMC, et al., 2020; FERNANDES MA, et al., 2018).

O estudo de Fernandes MA, et al. (2018), enfatizou sintomas relatados pelos universitários ao ingressar na universidade, sendo eles o nervosismo, sensação de estar assustado, indigestão ou desconforto abdominal, medo de que aconteça o pior, fadiga e irritabilidade. Além disso, Angelucci LT, et al. (2017), evidenciaram em sua pesquisa que os estilos de vida dos jovens não são tão saudáveis, e, apesar de estarem em boa saúde, manifestam certos comportamentos que podem influenciar negativamente sua saúde física e psicológica no futuro. No mais, todas essas evidências baseadas nos artigos selecionados, evidenciam que a vida acadêmica de fato interfere na saúde mental dos universitários.

\section{Ansiedade e depressão}

Aproximadamente 450 milhões de pessoas sofrem de perturbações mentais ou neurológicas no mundo. Por conseguinte, destaca-se a depressão, considerada a principal causa de incapacitação no mundo e com possibilidade de se tornar a segunda maior carga de doença até 2030. Em segundo lugar apresenta-se a ansiedade, comumente associada aos casos de depressão, nutrindo efeito importante na diminuição da qualidade de vida das pessoas (LEÃO AM, et al., 2018). 
A depressão é um transtorno multifatorial, pode surgir em qualquer período da vida e acarreta grande custo pessoal e social, aumentando o risco para comportamentos autodestrutivos, podendo, em último caso, levar ao suicídio (BARROSO SM, et al., 2019; LELIS KC, et al., 2020).

De acordo com o Manual Diagnóstico e Estatístico de Transtornos Mentais, DSM-IV-TR (American Psychological Associationm (APA), 2002), os principais sintomas depressivos caracterizam-se por humor deprimido na maior parte do dia, interesse ou prazer diminuído em todas ou quase todas as atividades, perda ou ganho significativo de peso sem estar em dieta, insônia ou hipersonia, agitação ou retardo psicomotor, fadiga ou perda de energia, sentimento de inutilidade ou culpa excessiva ou inadequada, capacidade diminuída de pensar ou concentrar-se, pensamentos de morte, ideação e tentativa de suicídio (CECCONELLO WW, et al., 2013; FERNANDES MA, et al., 2018; LEÃO AM, et al., 2018).

Já a ansiedade, está relacionada a ocorrência de acontecimentos em um dado momento, podendo gerar medo, apreensão, dúvida ou expectação, que causam diversos sintomas como, dores no peito, fadiga, palpitações, distúrbio do sono até consequentemente tornar-se patológica, se manifestando de forma mais frequente e intensa (LELIS KC, et al., 2020; LEÃO AM, et al., 2018; BARBOSA LNF, et al., 2020). Originando maior sofrimento psíquico.

Os motivos pelos quais a depressão e ansiedade estão entre os transtornos de humor mais comuns em estudantes universitários podem estar relacionados ao excesso de atividades acadêmicas, alta carga horária de aula e pensamentos inseguros relacionados a futura vida profissional (LELIS KC, et al., 2020).

A pesquisa de Flesch BD, et al. (2020) com 1865 estudantes ingressantes do primeiro semestre em uma universidade do sul do Brasil, concluiu que aspectos acadêmicos influenciam a ocorrência de depressão entre universitários. Corroborando com a pesquisa de Fernandes MA, et al. (2018), que em sua pesquisa com universitários obteve resultados três vezes maiores do índice de desenvolvimento de depressão quando comparados a população não universitária. Nesta pesquisa a prevalência dos sintomas de ansiedade e depressão foi bastante expressiva, respectivamente 62,9\% e 30,2\%.

Concomitante, Facioli AM, et al. (2020) também obteve resultado significativos, onde os níveis de depressão entre estudantes da instituição estudada estão acima dos índices observados na população geral, esses elevados índices estão fortemente associados com fatores acadêmicos. Similar ao estudo de Leão et al. (2018), em que a prevalência de depressão entre os estudantes foi de $28,6 \%$ e a de ansiedade foi de $36,1 \%$.

Vale ressaltar a forte relação entre sintomas depressivos e tentativas de suicídio, indicando uma insatisfação emocional. Essa insatisfação particularmente, quando ligada a sintomas depressivos, contribui para os índices de pensamentos e comportamentos de risco para o suicídio mais do que comumente é esperado. Outro fator relacionado a depressão e ansiedade é a solidão.

De acordo com Barroso SM, et al. (2019) a solidão é caracterizada por um profundo sentimento de isolamento social, podendo ser considerada como fator de risco para o desenvolvimento da depressão. Salienta ainda em seus estudos que, estudantes mais solitários apresentavam maior presença de problemas físicos e mentais, mostrando o impacto negativo da solidão nessa população. Considerando as estimativas da prevalência de transtornos mentais evidenciadas com base nos artigos selecionados, ressalta-se a importância da necessidade de atenção à saúde mental desta classe.

\section{Álcool e drogas}

O abuso de substâncias psicoativas é um fenômeno bastante antigo na história da humanidade. Estabelece um grave obstáculo à saúde pública, com sérias consequências pessoais e sociais no futuro dos jovens e de toda a sociedade. De acordo com Barbosa LNF, et al. (2020) compreende-se como substâncias psicoativas drogas que tem a capacidade de modificar a ordem funcional do cérebro, gerando alterações no estado intelecto/mental.

A Organização Mundial da Saúde (OMS) aponta que jovens de 18 a 25 anos tendem a apresentar comportamentos autodestrutivos, bem como abuso de álcool e drogas a fim de sinalizar carência de apoio e 
ajuda, já que por falta de conhecimento e autocompreensão, possuem dificuldades em pedir orientação corretamente (OSSE CMC e COSTA II, 2011). Sendo assim os alunos deveriam passar por uma diligência a fim de aprender uma visão qualificada da dimensão humana, para ajudar a enfrentar seus medos e ansiedades, podendo ajudar outros com os mesmos problemas (SANTOS SS, et al., 2013).

Segundo Sawicki WC, et al. (2018) ingerir bebida alcoólica de maneira exagerada pode causar problemas psicológicos na vida de seus consumidores. Os jovens estudantes, após entrarem no ensino superior, passam por mudanças sociais de grande impacto, uma vez que a maioria deles se deparam com uma independência que não possuíam antes. Essa liberdade os torna vulneráveis ao consumo desenfreado de substâncias psicoativas em geral.

Na pesquisa explicativa de Angelucci LT, et al. (2017) foi apontado que o uso de drogas tem maior influência na ansiedade e em check-ups médicos, sendo que sua saúde física também é piorada. Contudo, a relação do uso de drogas e a ansiedade é conjugada, uma vez que o uso rotineiro dessas substâncias pode levar ao desenvolvimento da ansiedade e a mesma pode levar ao maior consumo de drogas, o mesmo vale para outros transtornos mentais como depressão, anorexia e fobias sociais no geral (BARBOSA LNF, et al., 2020).

\section{Serviço de apoio acadêmico e psicológico}

De acordo com Leão AM, et al. (2018) a universidade deve proporcionar ao estudante uma formação completa, adequada e humanizada, de modo que esse universitário venha a ser atendido com base em suas necessidades pedagógicas e emocionais. Destarte, surge a importância de uma maior atenção a esses futuros profissionais, com enfoque na promoção da saúde, em especial a saúde mental, de forma a estarem emocionalmente bem mais preparados para o mercado de trabalho. Considerando a suma importância de identificar os alunos que apresentam maior risco de sofrer de problemas de saúde. (DAM CL, et al., 2014)

Em consonância a esta temática, Ariño DO e Bardagi MP (2018) salientam em seus estudos a necessidade de promover ações institucionais, que objetivem instrumentalizar os estudantes a gerenciar a vida acadêmica, preferencialmente no início do curso, a fim de melhorar o processo de formação superior, de modo que esse período seja vivenciado de maneira positiva, sem acarretar em sofrimento ou adoecimento de seus alunos.

As Instituições de ensino necessitam promover ações no ambiente institucional através de estratégias de controle e rastreamento para a saúde mental dos estudantes, para servir de base para acolhimento e intervenções a serem realizadas, com a finalidade de reduzir o sofrimento psicológico e melhorando a qualidade de vida desse grupo de indivíduos (LELIS KC, et al., 2020; FLESCH BD, et al., 2020)

Osse CMC e Costa II (2011) relataram em seus estudos que as instituições de ensino superior podem ofertar ajuda institucional, por meio de programas específicos de auxílio ao estudante, já que muitos alunos para se manter nela precisam de recursos para alimentação, transporte e até mesmo moradia, isso está ligado ao fato que de, muitos precisam se deslocar de sua cidade de origem para a cidade do Campus em que foi aprovado, e a Casa do Estudante Universitário é um dos recursos institucionais que pode ser disponibilizados para o universitário. Pois, cabe às instituições de ensino superior promover a criação de contextos que visem a integração total do indivíduo para a permanência com qualidade do estudante na universidade até a conclusão do curso.

\section{CONSIDERAÇÕES FINAIS}

O ensino superior impõe ao estudante uma série de desafios, a maioria não está preparada para a responsabilidade que o mundo acadêmico exige, essas situações geram desorientação, ansiedade, estresse, insônia, depressão, e tendências para o consumo de álcool e substâncias psicoativas, pois esses universitários são identificados como um grupo vulnerável e de risco nessas categorias. Portanto, a grande diversidade de situações clínicas que afetam esses estudantes são pontos fundamentais para 0 desenvolvimento de ações em saúde mental. Em resumo, é necessário que a instituição de ensino superior desenvolva estratégias para prevenir agravos a saúde mental de seus discentes, para que assim possam concluir o ensino superior com saúde mental estável. 


\section{REFERÊNCIAS}

1. ANGELUCCI LT, et al. Influência do estilo de vida, sexo, idade e bmi em saúde física e psicológica em estudantes universitários. Avanços na psicologia latino-americana, 2017; 35(3): 531-546.

2. ARIÑO DO, BARDAGI MP. Relação entre fatores acadêmicos e a saúde mental de estudantes universitários. Psicol. Pesqui., 2018; 12(3): 44-52.

3. BARBOSA LNF, et al. Ansiedade e depressão e uso de substâncias psicoativas em jovens universitários. SMAD, Rev. Eletrônica Saúde Mental Álcool Drog, 2020; 16(1): 1-8

4. BARROS S, CLARO HG. Processo ensino aprendizagem em saúde mental: o olhar do aluno sobre reabilitação psicossocial e cidadania. Rev Esc Enferm USP, 2011; 45(3):700-7.

5. BARROSO SM, et al. Solidão e Depressão: Relações com Características Pessoais e Hábitos de Vida em Universitários. Psicologia: Teoria e Pesquisa, 2019; 35, e35427.

6. BRAGA ALS. et al. Promoção à saúde mental dos estudantes universitários. Revista Pró-UniverSUS, 2017; 08(1): 48-54.

7. BRANDÃO MP, et al. Impacto da exposição acadêmica no estado de saúde dos estudantes universitários. Rev Saude Publica, 2011; 45 (1): 49-58.

8. CARDOSO HF, et al. Indicadores de Saúde Mental em Jovens: Fatores de Risco e de Proteção. Estudos Interdisciplinares em Psicologia, 2018; 9(3): 03-25.

9. CASTRO V R. Reflexões Sobre A Saúde Mental Do Estudante Universitário: Estudo Empírico Com Estudantes De Uma Instituição Pública De Ensino Superior. Ver, Gestão em Foco, 2017; 9: 380-401.

10. CECCONELLO WW, et al. Avaliação de sintomas depressivos e de fobia social em estudantes de graduação. Aletheia, 2013; 42: 71-81.

11. COSTA MC, MOREIRA YB. Saúde mental no contexto universitário. Seminários sobre Ensino de Design, 2016; 2(10):73-9.

12. DAM CL, et al. Sofrimento psicológico em estudantes universitários de ciências da saúde e sua relação com o envolvimento acadêmico. Rev esc enferm USP, 2014; 48(4): 715-722

13. FACIOLI AM, et al. Depressão entre estudantes de enfermagem e sua associação com a vida acadêmica. Revista Brasileira de Enfermagem, 2020; 73(1): e20180173.

14. FERNANDES MA, et al. Prevalência de sintomas ansiosos e depressivos em universitários de uma instituição pública. Revista Brasileira de Enfermagem [Internet], 2018; 71(5): 2298-394.

15. FLESCH BD, et al. Episódio depressivo maior entre universitários do sul do Brasil. Revista Saúde Pública, 2020; $54: 11$.

16. GOMES CFM, et al. Transtornos mentais comuns em estudantes universitários: abordagem epidemiológica sobre vulnerabilidades. SMAD, Rev. Eletrônica Saúde Mental Álcool Drog, 2020; 16(1): 1-8.

17. LEÃO AM, et al. Prevalência e fatores associados à depressão e ansiedade entre estudantes universitários da área da saúde de um grande centro urbano do Nordeste do Brasil. Revista Brasileira de Educação Médica, 2018; 42(4): 55-65.

18. LELIS KC, et al. Sintomas de depressão, ansiedade e uso de medicamentos em universitários. Revista Portuguesa de Enfermagem de Saúde Mental, 2020; (23): 09-14.

19. OSSE CMC, COSTA II. Saúde mental e qualidade de vida na moradia estudantil da Universidade de Brasília. Saúde Mental e Qualidade de Vida, 2011; 28(1): 115-122.

20. ROCHA AMC, et al. Tratamento Psíquico Prévio ao Ingresso na Universidade: Experiência de um Serviço de Apoio ao Estudante. Revista Brasileira de Educação Médica, 2020; 44 (3): e077.

21. SADOCK BJ, et al. Compêndio de Psiquiatria: Ciência do Comportamento e Psiquiatria Clínica. Porto Alegre: Artmed, 2016; 1490p.

22. SANTOS SS, et al. Atitudes, conhecimento e opiniões frente à saúde mental em alunos de graduação em enfermagem. Rev Esc Enferm USP, 2013; 47(5):1202-10.

23. SAWICKI WC, et al. Consumo de álcool, qualidade de vida e intervenção breve entre universitários de enfermagem. Rev. Bras. Enferm, 2018; 71(1): 505-12. 